Pacific Journal of Mathematics

POLYNOMIALS ORTHOGONAL OVER A DENUMERABLE SET 


\title{
POLYNOMIALS ORTHOGONAL OVER A DENUMERABLE SET
}

\author{
J. L. GOLDBERG
}

This paper concerns itself with characterizing the orthogonality domain and the distribution function for polynomials which satisfy

$$
\phi_{n+1}(x)=\left(x-a_{n}\right) \phi_{n}(x)-b_{n} \phi_{n-1}(x) \quad(n \geqq 0)
$$

with

$$
\phi_{-1}(x)=0 \text { and } \phi_{0}(x)=1
$$

under the restriction $a_{n}=0, b_{n}>0(n \geqq 0)$ and $\lim b_{n}=0$.

This extends the results of Dickinson, Pollak and Wannier [6] by replacing their restriction $\Sigma b_{n}<\infty$ with the weaker assumption $\lim b_{n}=0$, by correcting an apparent oversight, and by characterizing the distinction between the cases $\Sigma b_{n}<\infty$ and $\lim b_{n}=0$. In the course of this study we prove some theorems with occur rather naturally and seem of interest in their own right. Our approach owes its origins to ideas expressed in [4] and [6] and our techniques to the product and the series representations for a certain subclass of analytic functions studied by Richards [9] and related to functions characterized by a certain Stieltjes transform and continued fraction expansion.

More specifically; from a theorem of Favard [7] and Shohat [11], equations (1.1) and (1.2) and the assumptions $a_{n}$ real and $b_{n}>0(n \geqq 0)$ are sufflcient to imply that $\left\{\phi_{n}(x)\right\}$ is a real orthogonal set. Under the additional restrictions $a_{n}=0(n \geqq 0)$ and $\Sigma b_{n}<\infty$, Dickinson, Pollak and Wannier [6] have shown:

(i) The domain of orthogonality is a bounded denumerable set $S$, symmetric with respect to $x=0$, with $x=0$ the only non-isolated point.

(ii) The distribution function (unique, after normalization, because of the boundeness of $S$ ) with respect to which the polynomials $\left\{\phi_{n}(x)\right\}$ are orthogonal, is bounded, nondecreasing and with spectrum (the points of increase) the point set $S$ of (i). The points $S$ are the poles of a certain function, meromorphic in $1 / x$, whose residues are the values of the jumps of the distribution. (This statement appears to require modification because of the possibility of nonzero mass at $x=0$, a

Received June 12, 1964. Research supported in part by a grant from the National Science Foundation. 
point of $S$ not a pole. This oversight is examined in some detal below, see Theorem 3 and $\S 5$.)

(iii) The sequence $\left\{x^{n} \phi_{n}(x)\right\}$ converges to an entire function which is shown to be the denominator of the meromorphic function referred to in (ii).

Later, Carlitz [2], studing polynomials, remotely related to Laguerre polynomials, showed that properties (i) and (ii) hold for the polynomial sets (under a different normalization)

$$
g_{n+1}(x)=x g_{n}(x)-\frac{n}{(n+\gamma)(n+\gamma-1)} g_{n-1}(x) \quad(n \geqq 1)
$$

where $\gamma>0, g_{0}(x)=1$ and $g_{1}(x)=x$. For these polynomials $\sum b_{n}$ diverges and hence (Corollary 4, below) (iii) is false. Subsequently, Chihara [3, pg. 15] noted, and offered an independent proof of, a theorem implicit in the works of Stieltjes [13], equivalent to the proposition that $\lim b_{n}=0$ is necessary as well as sufflcient to insure a denumerable spectrum (with $x=0$ the only limit point) for the distribution relative to $\left\{\phi_{n}(x)\right\}, a_{n}=0, n=0,1, \cdots$.

In $\S 2$ we sketch the fundamental theorems of continued fractions and the theory of moments that are pertainent to our work. In $\S 3$ we prove the corrected generalization of the Dickinson, Pollak and Wannier theorem (Theorem 3) and set forth necessary and sufflcient conditions that $\lim x^{n} \phi_{n}(1 / x)$ be entire. Section 4 provides a representation theorem for the class of meromorphic functions relevent to our study and provides us with a means for investigating in $\S 6$, conditions under which mass at $x=0$ is not present. Finally we offer an example, due essentially to Wall [15] which explicitly contradicts (ii) (without the modification supplied in Theorem 3) but in which (iii) holds. The example is of interest independently of our use.

2. Preliminary theorems and notational conventions. We use this section to set forth those parts of the theory of continued fractions, theory of moments and theory of orthogonal polynomials which bear on the problems with which we wish to concern ourselves. None of these theorems are novel. They are stated in a form suitable for our purposes with their proofs outlined only in such detail that a specific reference may be quoted for their completion.

Consider the class of polynomial sets defined recursively by

$$
\phi_{n+1}^{(s)}(x)=x \phi_{n}^{(s)}(x)-b_{n+s} \phi_{n-1}^{(s)}(x)
$$

with

$$
\phi_{-1}^{(s)}(x)=0, \quad \phi_{0}^{(s)}(x)=1
$$


and

$$
b_{n}>0
$$$$
(n \geqq 0) \text {. }
$$

We write $\phi_{n}^{(0)}(x)$ as $\phi_{n}(x)$ and agree in general to omit all zero superscripts. We reserve the use of superscripts in parenthesis for nonnegative integers fixed in advance of any argument and never for use as a derivative.

It may be easily seen that $\left\{\phi_{n}^{(s)}(x)\right\}$ are the successive denominators and $\left\{\phi_{n-1}^{(s+1)}(x)\right\}$ the successive numerators (here our convention assures that $s$ is fixed and the sequences are indexed by $n$ ) of the convergents of

$$
\frac{1 \mid}{\mid 1}-\frac{b_{1+s} \mid}{\mid x}-\frac{b_{2+s} \mid}{\mid x}-\cdots-\frac{b_{n+s} \mid}{\mid x}-\cdots
$$

Such polynomial sequences have been studied by Dickison [4], [5], Dickinson, Pollak and Wannier [6], and perhaps most completely by Sherman [10], in which more references may be found. We pause to mention the important recursion relationship

$$
\phi_{n}^{(s)}(x)=x \phi_{n-1}^{(s+1)}(x)-b_{1+s} \phi_{n-2}^{(s+2)}(x) \quad(n \geqq 1),
$$

which follows from (2.4) but which may be proved independently by induction. If (2.5) is established first, one may observe directly that

$$
\frac{\phi_{n-1}^{(s+1)}(x)}{\phi_{n}^{(s)}(x)}=\frac{1 \mid}{\mid x}-\frac{b_{1+s} \mid}{\mid x}-\cdots-\frac{b_{n+s-1} \phi_{0}^{(n+s)}(x)}{\mid x},
$$

and hence the equivalence of (2.4) and $\lim _{n} \phi_{n-1}^{(s+1)}(x) / \phi_{n}^{(s)}(x)$. The definitions (2.1) and (2.2), the theorem of Favard-Shohat and the standard properties of real orthogonal polynomials lead to the observations which we state as

Lemma 1. All the zeros of the monic polynomials $\phi_{n}^{(s)}(x)$ are real and simple. The degree of $\phi_{n}^{(s)}(x)$ is precisely $n$ and $\phi_{n}^{(s)}(x)$ is an $\left(\begin{array}{c}\text { even } \\ \text { odd }\end{array}\right)$ function if $n$ is $\left(\begin{array}{c}\text { even } \\ o d d\end{array}\right)$. The zeros of $\phi_{n}^{(s)}(x)$ and $\phi_{n-1}^{(s+1)}(x)$ alternate; there is a zero of one polynomial separating two consecutive zeros of the other.

Next consider the sequence $\left\{\phi_{n-1}^{(s+1)}(x) / \phi_{n}^{(s)}(x)\right\}$. Set $z=1 / x$ in $(2.3)$ and define $G^{(s)}(z)$ by

$$
z G^{(s)}(z)=\frac{1 \mid}{\mid 1 / z}-\frac{b_{1+s} \mid}{\mid 1 / z}-\frac{b_{2+s} \mid}{\mid 1 / z}-\cdots-\frac{b_{n+s} \mid}{\mid 1 / z}-\cdots
$$

so that after and equivalence transformation 


$$
G^{(s)}(z)=\frac{1 \mid}{\mid 1}-\frac{b_{1+s} z^{2} \mid}{\mid 1}-\frac{b_{2+s} z^{2} \mid}{\mid 1}-\cdots-\frac{b_{n+s} z^{2} \mid}{\mid 1}-\cdots
$$

But a theorem of Stieltjes states: $A$ necessary and sufficient condition that (2.7) be a nonrational meromorphic function is $b_{n}>0$ and $\lim b_{n}=0$ (see Wall [14; Theorems 54.1 and 54.2]). Hence,

THeOREM 1. If $\left\{\phi_{n}^{(s)}(x)\right\}$ is defined by (2.1), (2.2) and (2.3) with $\lim b_{n}=0$, then for each nonnegative integer $s$.

$$
\lim _{n \rightarrow \infty} \frac{\phi_{n-1}^{(s+1)}(1 / z)}{z \phi_{n}^{(s)}(1 / z)}=G^{(s)}(z) .
$$

$G^{(s)}(z)$ is a transcendental meromorphic function and the convergence in (2.8) is uniform in compact sets which exclude the poles of $G^{(s)}(z)$.

As a corollary of this theorem we prove the properties of the orthogonality domain listed in (i) of the introduction. (See Chihara [3, pg. 15] for an alternate proof along different lines). Consider an interval $[c, d]$ free of poles of $G^{(s)}(1 / x)$. Lemma 1 assures that the zeros of $\phi_{n}^{(s)}(x)$ and $\phi_{n-1}^{(s+1)}(x)$ are not common. Hence, Theorem 1 and and Hurwitz's theorem imply that $[c, d]$ is ultimately free of zeros of $\phi_{n}^{(s)}(x)$. Thus any distribution function for $\left\{\phi_{n}^{(s)}(x)\right\}$ is constant in $[c, d]$, Szegö [12, Theorem 6.1.1]. But the poles of $G^{(s)}(1 / x)$ are a bounded set, symmetrically distributed with respect to $x=0$. Hence, we have proved:

Corollary 1. The orthogonality domain for the $\left\{\phi_{n}^{(s)}(x)\right\}$ of Theorem 1 is the bounded, denumerably infinite set of singularities of $G^{(s)}(1 / x)$. This set is isolated except at $x=0$, and symmetric with respect to the origin.

Suppose, by way of a converse, that $S$ is any bounded, denumerably infinite point set with $x=0$ the only limit point. Suppose the distribution function $\beta(x)$ (bounded and nondecreasing) has $S$ as its spectrum. We normalize $\beta(x)$ and all distribution function considered herein by specifying:

$$
\begin{aligned}
& \text { (i) } \int_{-\infty}^{\infty} d \beta=1 \\
& \text { (ii) } \beta(x)=\frac{1}{2}[\beta(x+0)+\beta(x-0)], \quad x \in S .
\end{aligned}
$$

Suppose further that $\beta(-x)=-\beta(x)$ (so that $S$ is symmetric) and that $\left\{p_{n}(x)\right\}$ is the unique set of monic polynomials orthogonal over $S$ with 
respect to $d \beta$. Then the symmetry of $d \beta$ leads directly to

$$
p_{n+1}(x)=x p_{n}(x)-B_{n} p_{n-1}(x)
$$

with $p_{-1}(x)=0$ and $p_{0}(x)=1$. This in turn defines the function (Szegö [12, Theorem 3.5.4])

$$
\begin{aligned}
F(z) & =\int_{-\infty}^{\infty} \frac{d \beta(t)}{1-t z} \\
& =\frac{1 \mid}{\mid 1}-\frac{B_{1} z^{2} \mid}{\mid 1}-\frac{B_{2} z^{2} \mid}{\mid 1}-\cdots
\end{aligned}
$$

It is now a consequence of the hypotheses on $\beta(x)$ that $F(z)$ is transcendental and meromorphic. Hence the theorem of Stieltjes mentioned prior to the statement of Theorem 1 yields $B_{n}>0, n=1,2, \cdots$ and $\lim B_{n}=0$. Thus,

COROLlary 2. A nondecreasing, symmetric distribution function, normalized by (2.9) having discrete, bounded spectrum with $x=0$ the only limit point, determines a transcendental meromorphic function with an expansion of the form (2.11), where $\left\{B_{n}\right\}$ is a positive null-sequence.

It is useful to have these two corollaries and Theorem 1 restated in somewhat different form.

COROLLARY 3. The denominators of the successive convergents of any continued fraction of the form (2.11) with $B_{n}>0(n>0)$ and $\lim B_{n}=0$ form a sequence of real orthogonal polynomials with the discrete domain of orthogonality described in Corollary 1.

Our proof of Theorem 3 (below) requires a Mittag-Leffler expansion of $G^{(s)}(z)$. To this end we call attension to the following theorem of Montel (Obrechkoff [8; Theorem XXI]):

If a sequence of rational functions converges uniformly to a meromorphic function and if the zeros and poles of each rational function are simple, real, and alternate, then the meromorphic function has the expansion

$$
B-A z+\sum_{n=1}^{\infty} A_{n}\left(\frac{1}{z-\alpha_{n}}+\frac{1}{\alpha_{n}}\right)
$$

where $\Sigma A_{n} / \alpha_{n}^{2}$ converges and $A, B, A_{1}, A_{2}, \cdots, A_{n}, \cdots$ have the same sign and the $\alpha_{n}$ are real and distinct. 
The hypotheses of this theorem are satisfied by the rational functions $\phi_{n-1}^{(s+1)}(1 / z) / \phi_{n}^{(s)}(1 / z)$ because of Lemma 1 and Theorem 1 . Furthermore, $G^{(s)}(z)$ is even. Hence, after simplification,

$$
G^{(s)}(z)=-A^{(s)}+\sum_{n=1}^{\infty} \frac{2 A_{n}^{(s)}}{z^{2}-\left(\alpha_{n}^{(s)}\right)^{2}} .
$$

Finally, $G^{(s)}(0)=1$ so that $A^{(s)} \leqq 0$ and $A_{n}^{(s)}<0(n>0)$. In this representation, and in all similar expansions, we agree to order the the poles, $0<\alpha_{1}^{(s)}<\alpha_{2}^{(s)}<\cdots, \alpha_{n} \rightarrow \infty$.

THEOREM 2. The transcendental meromorphic function of Theorem 1 has the expansion

$$
G^{(s)}(z)=-A^{(s)}+\sum_{n=1}^{\infty} \frac{2 A_{n}^{(s)}}{z^{2}-\left(\alpha_{n}^{(s)}\right)^{2}},
$$

where $-\Sigma A_{n}^{(s)}\left(\alpha_{n}^{(s)}\right)^{-2}<\infty, A^{(s)} \leqq 0$ and $A_{n}^{(s)}<0, n=1,2, \cdots$. The convergence is uniform in compact sets which exclude the poles of $G^{(s)}(z)$.

3. The construction of the distribution function. With the preliminaries now settled, we can proceed with a consideration of the first of our goals; namely, the construction which explicitly exhibts the relationship between $G^{(s)}(1 / x)$ and $\left\{\phi_{n}^{(s)}(x)\right\}$. We state this result as Theorem 3, a generalization and correction of the corresponding theorem in [6, Theorem 5]. The approach in this section owes its inspiration to the ideas expressed in [6].

Set $z=1 / x$ in (2.1), (2.2) and (2.5). Define $F_{n}^{(s)}(z)=z^{n} \phi_{n}^{(s)}((1 / z)$. Then,

$$
F_{n+1}^{(s)}(z)=F_{n}^{(s)}(z)-b_{n+s} z^{2} F_{n-1}^{(s)}(z) \quad(n \geqq 1)
$$

with

$$
F_{0}^{(s)}(z)=1, \quad F_{1}^{\prime s)}(z)=1
$$

and

$$
F_{n}^{(s)}(z)=F_{n-1}^{(s+1)}(z)-b_{1+s} z^{2} F_{n-2}^{(s+2)}(z) \quad(n \geqq 2) .
$$

Furthermore,

$$
\frac{\phi_{n-1}^{(s+1)}(1 / z)}{z \phi_{n}^{(s)}(1 / z)}=\frac{F_{n-1}^{(s+1)}(z)}{F_{n}^{(s)}(z)}
$$

Now divide (3.3) by $F_{n-1}^{(s+1)}(z)$ and let $n \rightarrow \infty$. This yields 


$$
\frac{1}{G^{(s)}(z)}=1-b_{1+s} z^{2} G^{(s+1)}(z)
$$

which may be interpreted as an alternate expression for (2.7). We combine (3.3) and (3.4) to obtain

$$
F_{n}^{(s)} G^{(s)}-F_{n-1}^{(s+1)}=b_{1+s} z^{2} G^{(s)}\left(F_{n+1}^{s+1} G^{(s+1)}-F_{n-2}^{(s+2)}\right)
$$

for $n \geqq 1$. (Here and in the next equation $F_{n}^{(s)}=F_{n}^{(s)}(z), G^{(s)}=G^{(s)}(z)$, etc.). Such an expression suggests iteration. With the aid of (3.4) written as

$$
\begin{aligned}
& F_{1}^{(s+n-1)} G^{(s+n-1)}-F_{0}^{(s \div n)}=G^{(n \div s-1)}-1 \\
& \quad=b_{n+s} z^{2} G_{0}^{(s+n)} G^{(n+s-1)}
\end{aligned}
$$

and after some simplication, including multiplying through by $z^{-n-p-1}$, we compute

$$
\begin{gathered}
z^{-n-p-1} G^{(s)}(z) F_{n}^{(s)}(z)-z^{-n-p-1} F_{n-1}^{(s+1)}(z) \\
=z^{n-p)-1} \prod_{k=1}^{n} b_{k+s} G^{(k+s)}(z) G^{(s)}(z)
\end{gathered}
$$

for any $p$ and all $n \geqq 1$. Now choose $C^{\prime}$ a circle small enough to exclude all the singularities of $G^{(s)}(z), G^{(s+1)}(z), \cdots, G^{(s+n)}(z)$. Such a circle exists because $G^{(m)}(0)=1$ for all $m$. The degree of $F_{k}^{(s)}(z)$ is $2[k / 2]$. Hence the residues at the origin of each term in (3.5) is readily computed and we have

$$
\frac{1}{2 \pi i} \int_{O^{\prime}} z^{-n-p-1} F_{n}^{(s)}(z) G^{(s)}(z) d z=\delta_{n p} \prod_{k=1}^{n} b_{k+s}
$$

for $0 \leqq p \leqq n$ and $n=1,2,3, \cdots$. If we define the empty product as unity, then (3.6) holds for $n=0$ also. The change of variables, $z=1 / x$, casts $(3.6)$ into

$$
\frac{1}{2 \pi i} \int_{\sigma} x^{p} \phi_{n}^{(s)}(x)\left\{x^{-1} G^{(s)}(1 / x)\right\} d x=\delta_{n p} \prod_{k=1}^{n} b_{k+s}
$$

$0 \leqq p \leqq n$ and $n \geqq 0$. Here $C$ is the circle reciprocal to $C^{\prime}$ 。 $C$ surrounds all of the singularities of $G^{(k+s)}(1 / x), k=0,1, \cdots, n$. The integration is taken in the positive direction. We may convert (3.7) into a real orthogonality relationship by substituting the representation of $G^{(s)}(z)$, given in Theorem 2, into (3.7) and interchanging the order of integration and summation. With the observation that the residue of $x^{-1} G^{(s)}(1 / x)$ at $x= \pm 1 / \alpha_{n}^{(s)}$ is $-A_{n}^{(s)}\left(\alpha_{n}^{(s)}\right)^{-2}>0$, we have

$$
-A^{(s)} \delta_{p 0} \varphi_{n}^{(s)}(0)+\Sigma x^{p} \phi_{n}^{(s)}(x) \operatorname{Res}\left\{x^{-1} G^{(s)}(1 / x)\right\}=\delta_{n p} \prod_{k=1}^{n} b_{k+s}
$$


for $0 \leqq p \leqq n, n=0,1,2, \cdots$. The summation is extended over all the poles of $x^{-1} G^{(s)}(1 / x)$. We express this and the results of section two as

THEOREM 3. Let $\left\{b_{n}\right\}$ be an arbitrary sequence of positive constants with $\lim b_{n}=0$. Suppose $\left\{\phi_{n}^{(s)}\right\}$ are the sets of orthogonal polynomials determined by (2.1) and (2.2). Suppose $G^{(s)}(z)$ is defined by (2.7) and $\beta^{(s)}$ is the unique normalized distribution function associated with $\left\{\phi_{n}^{(s)}(x)\right\}$. Then for each nonnegative integer $s$,

(i) the spectrum of $\beta^{(s)}$ is the closure of the set of poles of $G^{(s)}(1 / z) ;$ namely, $x=0$ and $x= \pm 1 / \alpha_{n}^{(s)}, n=1,2, \cdots$.

(ii) the jump of $\beta^{(s)}$ at these poles is equal to the residue of $x^{-1} G^{(s)}(1 / x)$ there. That is,

$$
\beta^{(s)}(x+0)-\beta^{(s)}(x-0)=-A_{n}^{(s)}\left(\alpha_{n}^{(s)}\right)^{-2}
$$

for $x= \pm 1 / \alpha_{n}^{(s)}$.

(iii) $\beta^{(s)}(+0)-\beta^{(s)}(-0)=-A^{(s)}$.

(iv) For each $p, 0 \leqq p \leqq n$ and all $n=0,12, \cdots$,

$$
\int_{-a}^{a} x^{p} \phi_{n}^{(s)}(x) d \beta^{(s)}=\int_{-a}^{a} \phi_{p}^{(s)}(x) \phi_{n}^{(s)}(x) d \beta^{(s)}=\hat{\delta}_{n p} \prod_{k=1}^{n} b_{k+s},
$$

where $[-a, a]$ is an interval large enough to include the bounded set, $\left\{ \pm 1 / \alpha_{n}^{(s)}\right\}$.

The criterion $\Sigma b_{n}<\infty$ is both necessary and sufficient to imply the existence of $\lim _{n} x^{n} \dot{\phi}_{n}^{(s)}(1 / x)$.

COROLLARY 4. If $\Sigma b_{n}<\infty$ then (uniformly)

$$
\lim _{n \rightarrow \infty} \frac{\phi_{n+1}^{(s+1)}(1 / x)}{x \phi_{n}^{(s)}(1 / x)}=\frac{\lim _{n \rightarrow \infty} x^{n-1} \phi_{n-1}^{(s+1)}(1 / x)}{\lim _{n \rightarrow \infty} x^{n} \phi_{n}^{(s)}(1 / x)}=\frac{E^{(s+1)}(x)}{E^{(s)}(x)}=G^{(s)}(x) .
$$

Here, $E^{(s+1)}(x)$ and $E^{(s)}(x)$ are entire functions. Conversely, if $\lim _{n} x^{n} \phi_{n}^{(s)}(1 / x)$ converges uniformly in a bounded closed domain about $x=0$ then $\Sigma b_{n}<\infty$.

Proof. The sufficiency, with the modification at $x=0$ previously mentioned, is the main theorem of Dickinson, Pollak and Wannier. Their proof depends only upon $\Sigma b_{i}<\infty$ and hence is applicable here. The necessity is proved by an appeal to a theorem of Polya (Obrechkoff, [8, Theorem IV] which states in eflect that the limit of a uniformly 
converging sequence of polynomials with real, symmetric zeros is an entire function. Therefore, the coefficient of $x^{2}$ in $x^{n} \dot{p}_{n}^{(s)}(1 / x)$ converges to the coefficient of $x^{2}$ in the series expansion of the limit function. But, we see that $x^{n} \phi_{n}^{(s)}(1 / x)=1-\left(b_{1+s}+b_{2+s}+\cdots+b_{n+s}\right) x^{2}+0\left(x^{4}\right)$ for $n=1,2, \cdots$. This proves the necessity. More interestingly;

THeOREM 4. A necessary and sufficient condition that $\lim x^{n} \phi_{n}^{(s)}(1 / x)$ converges uniformly in some bounded, closed domain containing $x=0$ (and hence converges to an entire function) is that $\Sigma \alpha_{n}^{-2}<\infty$.

Proof. Assume that $\lim _{n 2} x^{n} \phi_{n}^{(s)}(1 / x)$ converges uniformly. But the aforementioned theorem of Polya we know the limit function is entire. Denote its zeros by $\pm \alpha_{n}^{(s)}, 0<\alpha_{1}^{(s)}<\alpha_{2}^{(s)}<\cdots$. Let $\pm \alpha_{k, n}, k=$ $1,2, \cdots[n / 2]$ be the $2[n / 2]$ zeros of $x^{n} \phi_{n}^{(s)}(1 / x)$ ordered

$$
0<\alpha_{1, n}<\alpha_{2, n} \cdots<\alpha_{[n / 2], n} .
$$

Now a theorem of Hurwitz asserts that $\lim _{n} \alpha_{k, n}=\alpha_{k}^{(s)}, k=1,2, \cdots$. Referring once again to Polya's Theorem we conclude that $\Sigma\left(\alpha_{k}^{(s)}\right)^{-2}$ converges. Of course, the zeros $\pm \alpha_{k}^{(s)}$ are the poles of $G^{(s)}(1 / x)$. From (3.4) they are also the zeros of $G^{(s-1)}(1 / x)$. But the zeros and poles of $G^{(s-1)}(1 / x)$ (for any $s$ ) alternate on the real axis. Hence $\Sigma\left(\alpha_{k}^{(s-1)}\right)^{-2}$ also converges. Successive applications of this reasoning yields the convergence of $\Sigma\left(\alpha_{k}^{(0)}\right)^{-2}=\Sigma \alpha_{k}^{-2}$ after $s$ steps. We prove the sufficiency by showing that the convergence of $\Sigma \alpha_{k}^{-2}$ implies the convergence of $\Sigma b_{n}$. Towards this end we note that the zeros of $\phi_{n}^{(s)}(x)$ and $\phi_{n-1}^{(s+1)}(x)$ are interlaced (Lemma 1). In our notation, the reciprocals of these zeros are ordered as follows;

$$
\cdots<\alpha_{1, n+1}<\alpha_{1, n}^{\prime}<\alpha_{2, n+1}^{\prime}<\alpha_{2, n}<\cdots,
$$

for $n=1,2, \cdots$ Hence,

$$
\begin{gathered}
\frac{1}{\alpha_{1, n}^{2}}<\frac{1}{\alpha_{1, n+1}^{2}}<\frac{1}{\alpha_{1, n+2}^{2}}<\cdots \\
\frac{1}{\alpha_{2, n}^{2}}<\frac{1}{\alpha_{2, n+1}^{2}}<\frac{1}{\alpha_{2, n+2}^{2}}<\cdots \\
\vdots \quad \vdots \\
\frac{1}{\alpha_{r, n}^{2}}<\frac{1}{\alpha_{r, n+1}^{2}}<\frac{1}{\alpha_{r, n+2}^{2}}<\cdots
\end{gathered}
$$

for $r<[n / 2]$. Thus $\alpha_{r, n}^{-2}<\alpha_{r}^{-2}$. By hypothesis $\Sigma \alpha_{n}^{-2}<\infty$. Hence by Tannery's theorem (Browwich [1, pg. 136]).

$$
\lim _{n \rightarrow \infty} \sum_{k=1}^{[n / 2]} \alpha_{k, n}^{-2}=\sum_{k=1}^{\infty} \alpha_{k}^{-2}
$$


But $b_{1}+b_{2}+\cdots+b_{n}=\sum_{k=1}^{[n / 2]} \alpha_{k, n}^{-2}$, since the coefficient of $x^{2}$ in $x^{n} \phi_{n}^{(s)}(1 / x)$ is the sum of the squares of the zeros of $\phi_{n}^{(s)}(x)$. Corollary 4 completes the proof.

For a special case, Dickinson [4] has computed the moments, $\left\{m_{n}^{(s)}\right\}$, of $\beta^{(s)}$ in terms of the parameters of $G^{(s)}(z)$. We know in advance that the odd moments are zero (Shohat [11, Theorem II]) and that the moments are the coefficients in the Taylor series expansion of $G^{(s)}(z)$ about $z=0$. Specifically,

COROLLARY 5. Under the hypothesis of Theorem 3;

$$
\begin{aligned}
& m_{n}^{(s)}=0, \quad n \text { odd }, \\
& m_{n}^{(s)}=-A^{(s)}-2 \sum_{k=1}^{\infty} A_{k}^{(s)}\left(\alpha_{k}^{(s)}\right)^{-2}=1, \\
& m_{n}^{(s)}=-2 \sum_{k=0}^{\infty} A_{k}^{(s)}\left(\alpha_{k}^{(s)}\right)^{-n-2}, \quad n>0 \text { and even } .
\end{aligned}
$$

Proof. We have from Theorem 2 that

$$
\begin{aligned}
G^{(s)}(z) & =-A^{(s)}+\sum_{n=1}^{\infty} \frac{2 A_{n}^{(s)}}{z^{2}-\left(\alpha_{n}^{(s)}\right)^{2}} \\
& =-A^{(s)}-2 \sum_{n=1}^{\infty} A_{n}^{(s)}\left(\alpha_{n}^{(s)}\right)^{-2}\left\{1-z^{2} /\left(\alpha_{n}^{(s)}\right)^{2}\right\}^{-1} \\
& =-A^{(s)}-\sum_{k=0}^{\infty} z^{2 k} \sum_{n=1}^{\infty} 2 A_{n}^{(s)}\left(\alpha_{n}^{(s)}\right)^{-2 k-2}
\end{aligned}
$$

for $|z|<\left|\alpha_{1}^{(s)}\right|$. Thus

$$
x^{-1} G^{(s)}(1 / x)=-A^{(s)} / x-\sum_{k=0}^{\infty} x^{-2 k-1} \sum_{n=1}^{\infty} 2 A_{n}^{(s)}\left(\alpha_{n}^{(s)}\right)^{-2 k-2} .
$$

But then

$$
\frac{1}{2 \pi i} \int_{\sigma} x^{n}\left\{x^{-1} G^{(s)}(1 / x)\right\} d x=\int_{-a}^{a} x^{n} d \beta^{(s)}
$$

and the Corollary is proved.

4. A representation theorem for the meromorphic function $G^{(s)}(z)$. In previous sections we have concentrated on the determination of the spectrum of $\beta^{(s)}$ from a knowledge of $\left\{b_{n}\right\}$. In this and the succeeding section we direct our attention to the class of meromorphic functions which determine real orthogonal polynomials with distributions having spectra of the type described in Corollary 1 . We denote the class of such meromorphic functions by PIF; a notation motivated by the notation for a related class of functions. We express our main theorem by the following: 
THEOREM 5. The following four statements are equivalent*:

$$
\begin{aligned}
& z F(z) \in P I F \\
& F(z)=\frac{1 \mid}{\mid 1}-\frac{B_{1} z^{2} \mid}{\mid 1}-\frac{B_{2} z^{2} \mid}{\mid 1}-\cdots,
\end{aligned}
$$

with $B_{n}>0 n=1,2, \cdots$, and $\lim B_{n}=0$.

$$
F(z)=-A+\sum_{1}^{\infty} \frac{2 A_{n}}{z^{2}-\alpha_{n}^{2}},
$$

where $A \leqq 0, A_{n}<0, n=1,2,3, \cdots,-A-\Sigma 2 A_{n} \alpha_{n}^{-2}=1,0<\alpha_{1}<\alpha_{2}<\cdots$, $\alpha_{n} \rightarrow \infty$.

$$
F(z)=\prod_{n=1}^{\infty} \frac{1-z^{2} / \gamma_{n}^{2}}{1-z^{2} / \alpha_{n}^{2}}
$$

where $0<\alpha_{1}<\gamma_{1}<\alpha_{2}<\gamma_{2}<\cdots, \alpha_{n} \rightarrow \infty$ and $\prod_{n=1}^{\infty}\left(1+\gamma_{n}^{-2}\right)\left(1+\alpha_{n}^{-2}\right)^{-1}$ converges (and is therefore $\neq 0$ ).

Proof. $\quad(1) \Leftrightarrow(2)$ is established in $\S 2$ along with $(2) \Rightarrow 3$ (Theorem 2). We shall prove $(3) \Rightarrow(2)$ and then $(3) \Leftrightarrow(4)$ to complete the proof.

(a) We prove $(3) \Rightarrow(2)$. Suppose $F$ is defined by (3). Then $-A-\Sigma 2 A_{n} \alpha_{n}^{-2}=1$ establishes the uniform convergence of the righthand side of (3). Hence $F$ is transcendentally meromorphic and analytic at $z=0$. The Taylor series (in $z^{2}$ ) for $F$ at $z=0$ has only positive coefficients. From the theory of continued fractions (Shohat [11, pg. $455]$ ), we deduce a representation for $F$ in the form (2) with the stated conditions on $\left\{B_{n}\right\}$.

(b) To prove the equivalence of (3) and (4) we set $z=i t$ and define $f(t)=t F(i t)$. Then $f(t)$ is a meromorphic function which maps the right half-plane into itself, the imaginary axis into itself and the reals into the reals. These properties of $f(t)$ follow, if we assume $F$ is given by (3), by taking real and imaginary parts of (3). Richards [9] made a detailed study of such functions which he named iPRF ( $P R$ for positive real part; $P I$ for positive imaginary part in our case). The transformation, $t=-i z$ and the definition $F(z)=i f(-i z) / z$, therefore, converts Richards' theorems into results for $F(z)$. In particular then, $(3) \Rightarrow(4)$ as a consequence of [9; Theorem 12] and because $f^{\prime}(0)=$ $F^{\prime}(0)=1$. Conversely, if $F$ is given by $(4)$ then $f(t) \in i P R F$ by [9, Corollary 12.1] and $(4) \Rightarrow(3)$ by [9; Corollary 10.1]. This completes the proof.

5. The constants $A^{(s)}$. The mass assigned by the weight function

* We suspend our convention on superscripts for this section. 
to each point of the orthogonality domain may be determined, as we have seen, by examination of the residue at each pole of a function meromorphic in $1 / x$ with the single exception of the mass at zero, $-A^{(s)}$. If the mass at zero is zero for each $s$, as it is in the examples considered by Dickinson, Pollak and Wannier [6] and Carlitz [2], the exception is vacuous. It is of some interest then, to consider the problem of characterizing in function-theoretic terms those PIF functions with $A^{(s)}=0, s=0,1,2, \cdots$. In the course of this section we derive some theorems, parts of which yield conditions assuring nonzero mass at $x=0$. We begin by proving a Lemma fundamental to this part of our study.

Lemma 2. In the Stolz domain,

$$
0<\theta_{1} \leqq \arg z \leqq \theta_{2}<\pi, \quad \text { and for each } s \geqq 0 \text {, }
$$

$$
\lim _{|z| \rightarrow \infty} G^{(s)}(z)=-A^{(s)} \text {. }
$$

Proof. This is a well-known theorem in a different guise. For, [9, Theorem 5 and Corollary 10.1] shows that $\lim _{|z| \rightarrow \infty} f(t) / t$ exists $(t$ in the domain $|\arg t| \leqq \theta<\pi / 2)$ and is nonnegative. Now $G^{(s)}(z)$ is $P I F$ from Theorem 5 (2), so that $f(t)=t G^{(s)}(i t) \in I P R$ and the Lemma follows from [9; Corollary 10.1]. A second Lemma follows from (3.4) and Lemma 2.

LEMMA 3. Either the terms of $\left\{A^{(s)}\right\}_{s=1}^{\infty}$ are all zero or they are alternately zero and nonzero.

Proof. Set $z=i y,(y$ real $)$ in (3.4) and write

$$
\frac{1}{G^{(s)}(i y)}=1+b_{1+s} y^{2} G^{(s+1)}(i y) \text {. }
$$

Then one interation of (5.2) yields

$$
\frac{1}{G^{(s)}(i y)}=1+b_{1+s}\left(1 / y^{2}+b_{2+s} G^{(s+2}(i y)\right)^{-1} .
$$

Now let $y \rightarrow \infty$ in (5.2) and (5.3) and evoke Lemma 2. Equation (5.2) shows that $A^{(s)} A^{(s+1)} \neq 0$ is impossible and (5.3) shows that either $A^{(s)}=A^{(s+2)}=0$ or $A^{(s)} A^{(s+2)} \neq 0$ for every $s$. But this is just an alternate way of expressing the content of Lemma 3 .

THEOREM 6. For all $s=0,1,2, \cdots$

$$
-A^{(s)}=\lim _{n \rightarrow \infty} \prod_{1}^{n}\left(\alpha_{k}^{(s)} / \alpha_{k}^{(s+L)}\right)^{2}<\infty .
$$


Proof. Set $z=(i y)^{-1}$ in Theorem 5 and note that

$$
\lim _{|z| \rightarrow \infty} G^{(s)}(z)=-A^{(s)}=\lim _{y \rightarrow 0} \prod_{1}^{\infty}\left[\frac{y^{2}+\left(\alpha_{n}^{(s+1)}\right)^{-2}}{y^{2}+\left(\alpha_{n}^{(s)}\right)^{-2}}\right] \text {. }
$$

Since

$$
\frac{\left(\alpha_{n}^{(s)}\right)^{-2}-\left(\alpha_{n}^{(s+1)}\right)^{-2}}{y^{2}+\left(\alpha_{n}^{(s)}\right)^{-2}}<1-\left(\frac{\alpha_{n}^{(s)}}{\alpha_{n}^{(s+1)}}\right)^{2}
$$

for all $y$ and every $n$, the hypothesis that $\Pi\left(\alpha_{n}^{(s)} / \alpha_{n}^{(s+1)}\right)^{2}$ converges implies the uniform convergence of the rightmost factor in (5.5) in every set $y^{2} \leqq R^{2}$ and thus the continuity of $G^{(s)}(1 / i y)$ at $y=0$. This proves (5.4) when the product converges. Now $\left|\alpha_{n}^{(s)}\right|<\left|\alpha_{n}^{(s+1)}\right|$ so that divergence of $\Pi\left(\alpha_{n}^{(s)} / \alpha_{n}^{(s+1)}\right)^{2}$ is divergence to zero. Hence, given any $\varepsilon>0$ there exists an $N$ such that for all $n>N$

$$
\prod_{1}^{N}\left(\alpha_{n}^{(s)} / \alpha_{n}^{(s+1)}\right)^{2}<\varepsilon
$$

But

$$
\begin{aligned}
& \prod_{1}^{\infty}\left[\frac{y^{2}+\left(\alpha_{n}^{(s+1)}\right)^{-2}}{y^{2}+\left(\alpha_{n}^{(s)}\right)^{-2}}\right] \leqq \prod_{1}^{N}\left[\frac{y^{2}+\left(\alpha_{n}^{(s+1)}\right)^{-2}}{y^{2}+\left(\alpha_{n}^{(s)}\right)^{-2}}\right] \\
\leqq & \prod_{1}^{N}\left[\frac{y^{2}+\left(\alpha_{n}^{(s+1)}\right)^{-2}}{\left(\alpha_{n}^{(s)}\right)^{-2}}\right] \leqq\left(1+\frac{1}{N}\right)^{N} \prod_{1}^{N}\left(\alpha_{n}^{(s)} / \alpha_{n}^{(s+1)}\right)^{2} .
\end{aligned}
$$

The leftmost inequality holds for every $N$ because each term in the product is less than one for every $y$. The last inequality holds for all $y$ satisfying $y \leqq\left(\sqrt{N} \alpha_{n}^{(s+1)}\right)^{-1} \quad n=0,1, \cdots, N$. Because of the ordering of the poles of $G^{(s)}(z)$, this can be accomplished by the restriction $y \leqq\left(\sqrt{N} \alpha_{N}^{(s+1)}\right)^{-1}$. Hence the limit on the right side of (5.5) must be zero, proving Theorem 6 if the product diverges. A less complete result follows from (2.13).

THEOREM 7. If $A^{(s+1)}=0$ then a necessary and sufficient condition for $A^{(s)} \neq 0$ is that $\Sigma A_{n}^{(s+1)}$ converge. In either case

$$
A^{(s)}=-\left(1-b_{1+s} \Sigma 2 A_{n}^{(s+1)}\right)^{-1}
$$

(suitably interpreted if $\Sigma A_{n}^{(s)}$ diverges).

Proof. Set $z=(i y)^{-1}\left(y\right.$ real) in the representation for $G^{(s+1)}$ given by (2.13). Since $A^{(s+1)}=0$ and $0<\alpha_{1}^{(s+1)}<\alpha_{2}^{(s+1)}<\cdots$, we have for all $y \leqq\left(\alpha_{1}^{(s+1)}\right)^{2}$,

$$
(-1 / 2) \sum_{1}^{N} A_{n}^{(s+1)} \leqq \frac{-A_{n}^{(s+1)}}{1+\left(\alpha_{1}^{(s+1)}\right)^{-2} y^{2}} \leqq \sum_{1}^{N} \frac{-A_{n}^{(s+1)}}{1+\left(\alpha_{n}^{(s+1)}\right)^{-2} y^{2}}
$$




$$
\leqq \sum_{1}^{\infty} \frac{-A_{n}^{(s+1)}}{1+\left(\alpha_{n}^{(s+1)}\right)^{-2} y^{2}}=y^{-2} G^{(s+1)}(-i / y)
$$

But from (3.4), with $z=(i y)^{-1}$,

$$
y^{-2} G^{(s+1)}(-i / y)=b_{1+s}^{-1}\left(\frac{1}{G^{(s)}(-i / y)}-1\right) .
$$

Let $y \rightarrow 0$ and assume $\Sigma A_{n}^{(s+1)}$ diverges. Then

$$
\lim _{y \rightarrow 0} G^{(s)}(-i / y)=-A^{(s)}=0 .
$$

Now suppose $\Sigma A_{n}^{(s+1)}$ converges. Since

$$
y^{-2} G^{(s+1)}(-i / y)=\sum_{1}^{\infty} \frac{-A_{n}^{(s+1)}}{1+\left(\alpha_{n}^{(s+1)}\right)^{-2} y^{2}} \leqq \sum_{1}^{\infty}-A_{n}^{(s+1)},
$$

we conclude that $\Sigma A_{n}^{(s+1)}\left[1+\left(\alpha_{n}^{(s+1)}\right)^{2} y^{2}\right]^{-1}$ converges uniformly for all $y$ in, say $y^{2} \leqq R$, and hence represents a continuous function at $y=0$. Therefore, from (5.6) and (5.7).

$$
b_{1+s}^{-1}\left(-1 / A^{(s)}-1\right)=-\sum_{1}^{\infty} A_{n}^{(s+1)} .
$$

This completes the proof.

Hence if $\left\{A^{(s)}\right\}$ is a sequence of zeros, $\sum A_{n}^{(s)}$ must diverge for each $s$. Conversely, if all such series diverge $\left\{A^{(s)}\right\}$ is a sequence of zeros. Is it possible for $\Sigma A_{n}^{(s)}$ to converge and $A^{(s)} \neq 0$ ? $A^{(s)}=0$ ? In other words, if $\left\{A^{(s)}\right\}$ is alternately zero and nonzero, what can be said about the convergence of $\Sigma A_{n}, \Sigma A_{n}^{(1)}, \Sigma A_{n}^{(2)}, \cdots$, besides the statement that they all cannot diverge? We leave this question open.

Finally,

THEOREM 8. The constants $\left\{b_{n+s}\right\}$ and $A^{(s)}$ are related by

$$
\frac{-1}{A^{(s)}}=1+\frac{b_{1+s}}{b_{2+s}}+\frac{b_{1+s} b_{3+s}}{b_{2+s} b_{4+s}}+\frac{b_{1+s} b_{3+s} b_{5+s}}{b_{2+s} b_{4+s} b_{6+s}}+\cdots,
$$

so that $A^{(s)}=0$ if and only if the series (5.8) diverges.

This theorem is known (see [3; Theorem 2] and the references therein). We include the statement here for completeness.

6. An example. Wall [15] studied a certain continued fraction which arose in a number-theoretic context. By suitable changes of variable this example may be used to illustrate the theorems of the 
previous sections. There seem to be relatively few cases of interesting special functions for which the sequences $\left\{b_{n}\right\}$ and $\left\{A_{n}^{(s)}\right\}$ are known explicitly and $\left\{A^{(s)}\right\}$ is not all zeros. The author was able to find only this one example. Choose $0<r<1$ and $0<q<1$ and define $b_{2}=r$,

$$
b_{2 k+2}=\left(1-r q^{k}\right) q^{k+1}, b_{2 k+3}=r q^{k+1}\left(1-q^{k+1}\right)
$$

$k=0,1,2, \cdots$;

$$
\begin{aligned}
& M_{1}=r \prod_{k=1}^{\infty}\left(1-r q^{k}\right) ; \\
& M_{k}=\frac{r^{k-1} M_{1}}{(1-q)\left(1-q^{2}\right) \cdots\left(1-q^{k-1}\right)},
\end{aligned}
$$

Then Wall [15] has shown, in our notation,

$$
\frac{1}{G^{(0)}(z)}=1+z^{2} \sum_{k=1}^{\infty} \frac{M_{k} q^{-k}}{z^{2}-q^{-k}}
$$

From (3.4) and (6.4) we deduce that

$$
G^{(1)}(z)=\sum_{k=1}^{\infty} \frac{-M_{k}\left(r q^{k}\right)^{-1}}{z^{2}-q^{-k}}
$$

so that $A^{(1)}=0,-2 A_{k}^{(1)}=M_{k}\left(r q^{k}\right)^{-1}$ and $\left(\alpha_{k}^{(1)}\right)^{2}=q^{-k}$. We use Theorem 7 with $s=0$ to deduce that $A^{(0)} \neq 0$ if $\Sigma M_{k}\left(r q^{k}\right)^{-1}$ converges. The ratio test yields the convergence of this series if $r<q$ and its divergence if $q<r$. Thus the terms in $\left\{A^{(s)}\right\}$ are all zero if $q<r$ and are alternately zero and nonzero, $A^{(0)} \neq 0$, if $r<q$. Wall has also shown that

$$
G^{(1)}(z)=\sum_{n=0}^{\infty}\left[q^{n} \prod_{k=0}^{n-1}\left(1-r q^{k}\right)\right] z^{2 n} .
$$

Hence, the moments $m_{2 n}^{(1)}$ can be read off immediately (see Corollary 5)

$$
m_{2 n}^{(1)}=q^{n} \prod_{k=0}^{n-1}\left(1-r q^{k}\right)
$$

where the empty product is defined as unity. Incidentally, $\Sigma b_{k}<\infty$ for all choices of $r$ and $q$ so that this example is one that is included in the Dickinson, Pollak and Wannier theory.

7. Acknowledgement. A special note of thanks is due Professor J.L. Ullman for the many hours of valuable discussions on the material of this note. His encouragement, interest and stimulation were a major source of inspiration. 


\section{REFERENCES}

1. T.J.I'a. Bromwich, An introduction to the theory of infinite series, Macmillan and Co. 1959.

2. L. Carlitz, On some polynomials of Tricomi, Boll. Unione Mat. Ital. (3) 13 (1958), $58-64$.

3. T.S. Chihara, Chain sequences and orthogonal polynomials, Trans. Amer. Math. Soc. 104 (1962), 1-16.

4. D.J. Dickinson, On Bessel and Lommel polynomials, Proc. Amer. Math. Soc. 5 (1954), 946-956.

5. - On certain polynomials associated with orthogonal polynomials, Boll. Unione Mat. Ital. (3), vol. 13, pp. 116-124.

6. D.J. Dickinson, H.O. Pollak, G.H. Wannier, On a class of polynomials orthogonal over a denumerable set, Pacific J. Math. 6 (1956), 239-247.

7. J. Favard, Sur les polynomes de Tchebicheff, C.R. Acad. Sci. Paris, 200 (1935), 2052-2053.

8. N. Obrechkoff, Quelques classes de fonctiones entières limites de polynomes et de fonctiones mèromerphes limites de fractions rationalles, Actualities Scientifiques et Industrielles, 891, Hermann et $\mathrm{C}^{i e}$, Paris, 1941.

9. P. Richards, A special class of functions with positive real part in a half-plane, Duke Math. J. 14 (1947), 777-786.

10. J. Sherman, On the numerators of the convergents of the Stieltjes continued fraction, Trans. Amer. Math. Soc. 35 (1933), 64-87.

11. J. Shohat, The relation of the classical orthogonal polynomials to the polynomials of Appell, Amer. J. Math. 58 (1936), 453-464.

12. G. Szegö, Orthogonal polynomials, Amer. Math. Soc. Colloquium Publication, vol. 23, New York, 1939.

13. T.S. Stieltjes, Recherches sur les fractions continues, Oeuvres, Tome II, Noordhoff, Groningen 1918 pp. 402-566.

14. H.S. Wall, Analytic theory of continued fractions, D. VanNostrand, 1948.

15. - A continued fraction related to some partition formulas of Euler, Amer. Math. Monthly, 48 (1941), 102-108. 


\title{
PACIFIC JOURNAL OF MATHEMATICS
}

\author{
EDITORS
}

\author{
H. SAmelson \\ Stanford University \\ Stanford, California \\ R. M. Blumenthal \\ University of Washington \\ Seattle, Washington 98105
}

\author{
J. DugundjI \\ University of Southern California \\ Los Angeles, California 90007
}

*Richard Arens

University of California

Los Angeles, California 90024

\section{ASSOCIATE EDITORS}
E. F. BECKENBACH
B. H. NeUmanN
F. WOLF
K. YoSIDA

\section{SUPPORTING INSTITUTIONS}

\author{
UNIVERSITY OF BRITISH COLUMBIA \\ CALIFORNIA INSTITUTE OF TECHNOLOGY \\ UNIVERSITY OF CALIFORNIA \\ MONTANA STATE UNIVERSITY \\ UNIVERSITY OF NEVADA \\ NEW MEXICO STATE UNIVERSITY \\ OREGON STATE UNIVERSITY \\ UNIVERSITY OF OREGON \\ OSAKA UNIVERSITY \\ UNIVERSITY OF SOUTHERN CALIFORNIA
}

\author{
STANFORD UNIVERSITY \\ UNIVERSITY OF TOKYO \\ UNIVERSITY OF UTAH \\ WASHINGTON STATE UNIVERSITY \\ UNIVERSITY OF WASHINGTON \\ * * * * \\ AMERICAN MATHEMATICAL SOCIETY \\ CALIFORNIA RESEARCH CORPORATION \\ SPACE TECHNOLOGY LABORATORIES \\ NAVAL ORDNANCE TEST STATION
}

Mathematical papers intended for publication in the Pacific Journal of Mathematics should by typewritten (double spaced). The first paragraph or two must be capable of being used separately as a synopsis of the entire paper. It should not contain references to the bibliography. No separate author's resumé is required. Manuscripts may be sent to any one of the four editors. All other communications to the editors should be addressed to the managing editor, Richard Arens, at the University of California, Los Angeles, California 90024.

50 reprints per author of each article are furnished free of charge; additional copies may be obtained at cost in multiples of 50 .

The Pacific Journal of Mathematics is published quarterly, in March, June, September, and December. Effective with Volume 13 the price per volume (4 numbers) is $\$ 18.00$; single issues, $\$ 5.00$. Special price for current issues to individual faculty members of supporting institutions and to individual members of the American Mathematical Society: $\$ 8.00$ per volume; single issues $\$ 2.50$. Back numbers are available.

Subscriptions, orders for back numbers, and changes of address should be sent to Pacific Journal of Mathematics, 103 Highland Boulevard, Berkeley 8, California.

Printed at Kokusai Bunken Insatsusha (International Academic Printing Co., Ltd.), No. 6, 2-chome, Fujimi-cho, Chiyoda-ku, Tokyo, Japan.

PUBLISHED BY PACIFIC JOURNAL OF MATHEMATICS, A NON-PROFIT CORPORATION

The Supporting Institutions listed above contribute to the cost of publication of this Journal, but they are not owners or publishers and have no responsibility for its content or policies.

* Basil Gordon, Acting Managing Editor until February 1, 1966. 


\section{Pacific Journal of Mathematics}

\section{Vol. 15, No. $4 \quad$ December, 1965}

Robert James Blattner, Group extension representations and the structure space ........... 1101

Glen Eugene Bredon, On the continuous image of a singular chain complex .............. 1115

David Hilding Carlson, On real eigenvalues of complex matrices .................... 1119

Hsin Chu, Fixed points in a transformation group ............................. 1131

Howard Benton Curtis, Jr., The uniformizing function for certain simply connected Riemann

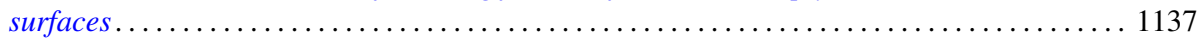

George Wesley Day, Free complete extensions of Boolean algebras................... 1145

Edward George Effros, The Borel space of von Neumann algebras on a separable Hilbert

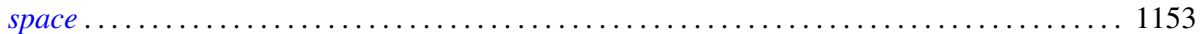

Michel Mendès France, $A$ set of nonnormal numbers ......................... 1165

Jack L. Goldberg, Polynomials orthogonal over a denumerable set ................ 1171

Frederick Paul Greenleaf, Norm decreasing homomorphisms of group algebras . . . . . . . . 1187

Fletcher Gross, The 2-length of a finite solvable group ........................ 1221

Kenneth Myron Hoffman and Arlan Bruce Ramsay, Algebras of bounded sequences ........ 1239

James Patrick Jans, Some aspects of torsion . . . . . . . . . . . . . . . . . . . . . . . 1249

Laura Ketchum Kodama, Boundary measures of analytic differentials and uniform

approximation on a Riemann surface ............................... 1261

Alan G. Konheim and Benjamin Weiss, Functions which operate on characteristic

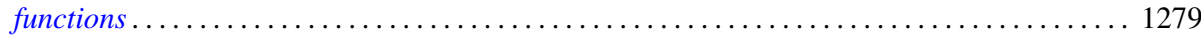

Ronald John Larsen, Almost invariant measures ............................ 1295

You-Feng Lin, Generalized character semigroups: The Schwarz decomposition ............ 1307

Justin Thomas Lloyd, Representations of lattice-ordered groups having a basis . . . . . . . . 1313

Thomas Graham McLaughlin, On relative coimmunity ....................... 1319

Mitsuru Nakai, $\Phi$-bounded harmonic functions and classification of Riemann surfaces ....... 1329

L. G. Novoa, On n-ordered sets and order completeness ..................... 1337

Fredos Papangelou, Some considerations on convergence in abelian lattice-groups . . . . . . . 1347

Frank Albert Raymond, Some remarks on the coefficients used in the theory of homology

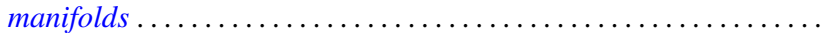

John R. Ringrose, On sub-algebras of a $C^{*}$-algebra .

Jack Max Robertson, Some topological properties of certain spaces of differentiable

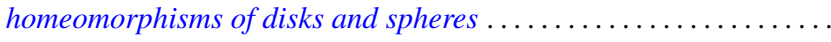

Zalman Rubinstein, Some results in the location of zeros of polynomials

Arthur Argyle Sagle, On simple algebras obtained from homogeneous general Lie triple systems. . . .

Hans Samelson, On small maps of manifolds ............................... 1401



Edsel Ford Stiel, Isometric immersions of manifolds of nonnegative constant sectional curvature

Earl J. Taft, Invariant splitting in Jordan and alternative algebras ................. 1421

L. E. Ward, On a conjecture of R. J. Koch . . . . . . . . . . . . . . . . . . . . . . . . . . . 1429

Neil Marchand Wigley, Development of the mapping function at a corner . . . . . . . . . . 1435

Horace C. Wiser, Embedding a circle of trees in the plane ....................... 1463

Adil Mohamed Yaqub, Ring-logics and residue class rings . . . . . . . . . . . . . . . . 1465

John W. Lamperti and Patrick Colonel Suppes, Correction to: Chains of infinite order and their application to learning theory ........................................ 1471

Charles Vernon Coffman, Correction to: Non-linear differential equations on cones in Banach

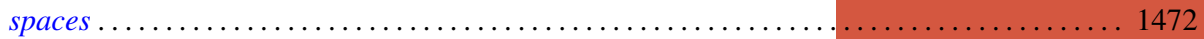

P. H. Doyle, III, Correction to: A sufficient condition that an $\operatorname{arc}$ in $S^{n}$ be cellular. . . . . . . . 1474

P. P. Saworotnow, Correction to: On continuity of multiplication in a complemented algebra 